\title{
Screening for minimal hepatic encephalopathy among asymptomatic drivers with chronic liver disease
}

\author{
Mohammad Eltaher Abdelrahman ${ }^{\mathrm{a}}$, Saad Zaky Mahmoud ${ }^{\mathrm{a}}$, Anwar M. Alib, \\ Haitham Ahmed Abdalla T. El-Khateeb ${ }^{\mathrm{c}}$, Ghada A. Mohamed ${ }^{\mathrm{d}}$
}

\begin{abstract}
Departments of ${ }^{\mathrm{a}}$ Tropical Medicine and Gastroenterology, ${ }^{b}$ Neurology, Faculty of Medicine, Assiut University, 'Department of Tropical Medicine and Gastroenterology, Police Hospital, ${ }^{\mathrm{d} D e p a r t m e n t}$ of Internal Medicine, Faculty of Medicine, Assiut University, Assiut, Egypt
\end{abstract}

Correspondence to Ghada A Mohamed, MD, Department of Internal Medicine, Assiut Faculty of Medicine, Assiut University, 71511, Egypt. Tel: 01004301177

e-mail: ghadaa1980@yahoo.com

Received 8 January 2018

Accepted 4 September 2018

The Egyptian Journal of Internal Medicine 2018, 30:217-222

\begin{abstract}
Background and aims
Minimal hepatic encephalopathy (MHE) may impair driving performance and adversely disturbs quality of life. Most drivers with chronic liver disease are not routinely screened for MHE and stay untreated due to the deficiency of standardization of normal values, simple tools, and skills to carry out tests. This study evaluated the usefulness of psychometric tests and critical flicker frequency (CFF) to diagnose MHE among screened drivers with chronic liver disease in Assiut, a city in Upper Egypt.

\section{Patients and methods}

A total of 100 drivers with chronic liver disease were screened for MHE. Routine investigations were carried out for all. Psychometric tests including number connection tests A, symbol digits test and CFF testing were applied for them. mini mental state examination questionnaires and Beck's inventory were carried out for those diagnosed as having MHE.

Results

MHE was found among 40\%, with $20(50 \%)$ in Child's A, $11(27.5 \%)$ in Child's B, and nine $(22.5 \%)$ in Child's $C$ cirrhosis $(P=0.027)$, and $45 \%$ of those with MHE had bad driving history. Twelve (12\%) patients had abnormal psychometric tests. The sensitivity and specificity of receiver operating characteristic curves for CFF in the diagnosis of MHE was $100 \%$, with a cutoff of less than or equal to $38.5 \mathrm{~Hz}$, and the area under the curve was 1.00 (95\% confidence interval, 0.964-1.00) $(P<0.0001)$. There was significant reverse correlation between CFF and Child-Pugh score $(r=-0.271, P=0.030)$.

\section{Conclusion}

Our data revealed a high prevalence of MHE (40\%) among drivers with liver cirrhosis. The CFF test is a simple, accurate, and reliable test for diagnosis of $\mathrm{MHE}$, with no age or literacy requirement. Therefore, we suggest to include the CFF test in screening of drivers with chronic liver disease for early discovery and proper management.
\end{abstract}

\section{Keywords:}

chronic liver disease, critical flicker frequency, minimal hepatic encephalopathy

Egypt J Intern Med 30:217-222

(c) 2019 The Egyptian Journal of Internal Medicine

1110-7782

\section{Introduction}

Minimal hepatic encephalopathy (MHE) is a subtle form of hepatic encephalopathy (HE); it is associated with increased mortality, risk of hospitalization, impairment of quality of life, and caregiver burden. MHE detection can guide treatment, which has the potential to improve outcomes and quality of life [1]. MHE is defined as the presence of at least one abnormal psychometric test and/or an abnormal slowing of the electroencephalogram [2].

Driving is a potentially dangerous and complex mission that integrates visuomotor coordination and selective attention. The impact of MHE on driving ability dates back to the early 1980s when Schomerus et al. [3] has subjected patients with cirrhosis to psychometric tests and driving tests; his study revealed that a significant number of MHE patients were unfit to drive. Since then, further studies have been carried out, and there is growing evidence of compromised driving abilities in cirrhotic patients who meet the criteria for $\mathrm{MHE}$ diagnosis.

Despite the impact of MHE, most cirrhotic patients are not systematically tested for MHE and remain untreated, due to the lack of normal values' standardization, simple tools, and expertise to administer the tests [4]. International consensus meetings recommended the use of psychometric

This is an open access journal, and articles are distributed under the terms of the Creative Commons Attribution-NonCommercial-ShareAlike 4.0 License, which allows others to remix, tweak, and build upon the work non-commercially, as long as appropriate credit is given and the new creations are licensed under the identical terms. 
testing for early detection of MHE [5,6]. Psychometric testing is a reliable tool for the diagnosis of $\mathrm{MHE}$ because it can detect the majority of these psychometric disorders related to MHE. The weakness of this tool is the need for data on the level of education [7].

Critical flicker frequency (CFF) is a well-recognized neurophysiological technique that measures the ability of the central nervous system to identify flickering light and is influenced directly by cortical activity [8]. Therefore, CFF could help to detect certain characteristics of MHE that would be misdiagnosed by psychometric testing, thereby pre-empting the disadvantage associated with neurophysiological tools. CFF is reliable, simple, easy to apply, more sensitive, and is not affected by age or level of education. The altered CFF predicts the risk for the development of the manifest $\mathrm{HE}$ but not survival. More than two-thirds of patients at Child-Pugh's Class $\mathrm{B}$ or $\mathrm{C}$ were noticed to be at risk of developing a manifest $\mathrm{HE}$ in the first year of followup when CFF was impaired. Contrarily, only $4 \%$ of patients without psychometric testing and/or CFF alterations developed $\mathrm{HE}$.

\section{Aim}

The high prevalence of chronic liver disease and motor car accidents in Upper Egypt, motivated us to screen for MHE among drivers with chronic liver disease, by using two validated techniques, psychometric tests [symbol digits test (SDT) and number connection tests A (NCT-A)] and CFF, after the drivers undergoing the mini mental scale examination.

\section{Patients and methods}

\section{Patients}

Our case-control study consisted of 100 consecutive drivers who presented to and were followed-up at the Outpatient Clinic of Tropical Medicine and Gastroenterology at the Assuit University Hospital. According to ethical committee of faculty of medicine Assiut university, all patients recruited in the study were signed written consent and informed by all steps in the study. All patients were seropositive for viral markers and were found to be cirrhotic on the basis of clinical, biochemical, and ultrasound data. Another group of patients $(N=30)$ who were age and sex matched was included as a control group. The exclusion criteria were as follows: manifest $\mathrm{HE}$ or a history of overt $\mathrm{HE}$, recent history of alcohol intake, use of psychotropic drugs or interferons, use of benzodiazepines, current intake of antibiotics for gastrointestinal bleeding or history for drug intake for epilepsy, renal insufficiency, intrahepatic transjugular portosystemic shunt for portal hypertension, diabetes mellitus, hepatocellular carcinoma, congestive heart failure, pulmonary disease, acute infection, and neurological or psychiatric disorders that may affect the test outcomes.

\section{Methods}

History and laboratory investigation

For each patient in this study, the following data were collected: complete demographic data, age and sex, alcohol consumption (quantity and type), smoking (type and frequency of smoking per day), level of education, occupation, residential area and selfreported driving history, a complete history of liver disease, duration of illness, neurological symptoms (insomnia, sleep disorders, memory impairment, bizarre behavior, drowsiness, confusion, tremors, and irritability), and history of HE. A complete clinical examination was performed for all patients, including a general examination, with particular attention to the manifestations of hepatic impairment and liver encephalopathy. Venous blood samples were also collected for liver function tests and markers of viral hepatitis. An abdominal ultrasound has been established to investigate the stigmata of chronic liver disease.

\section{Psychometric assessment}

(1) SDT: This test was used to evaluate the motor speed and accuracy wherein drivers were given a list of numbers from one to nine accompanied with symbols and were asked to fill blanks with symbols corresponding to each number. An initial demonstration was presented for each participant to be familiar with the test. Test results are the total number of correctly paired symbols to the number within a $90 \mathrm{~s}$ period, wherein a high value $(>90 \mathrm{~s})$ indicates poor performance [9].

(2) NCT-A: This test is a derivative of the Trail Making Test; an Egyptian version was used to assess cognitive motor abilities, wherein all participants were asked to link the written numbers in the paper consecutively from 1 to 25 as fast as possible, after clarification was carried out to ensure sufficient understanding and performance by the participants. Test results included the time required for testing and error correction, wherein a high value $(>78 \mathrm{~s})$ indicated poor performance [6].

(3) CFF: CFF is a test of the functional efficiency of the cortex, which has a direct relationship with psychometric abnormalities. In this test, the 
patient shows the minimum frequency at which a blinking light is always observed as flickering and unfused, using a portable device (Hepato-Norm analyzer, Merz Company, Germany). The gradual increase in the frequency of light from $25 \mathrm{~Hz}$ makes it possible to determine the melting frequency threshold, which is the frequency at which the blinking light is perceived by the patient as a fused light. On the contrary, a gradual decrease in the frequency of the light pulse from $60 \mathrm{~Hz}$ downwards is also carried out, and the CFF threshold is determined as the frequency when the impression of fused light has changed into a flicker. The CFF is considered abnormal when the value is less than $39 \mathrm{~Hz}[10]$.

The psychometric tests and CFF were carried out by all the participants assisted by the medical personnel without knowledge of the diagnosis. The diagnosis of MHE was established when either SDT or both SDT and NCT were abnormal. Beck's inventory and questionnaires of mini mental status examination were used for those who were established as having MHE in order to exclude depression by the first and any related medical illness by the latter. For the psychometric tests as well as CFF test, informed consent was made within the same framework.

\section{Statistical analysis}

Data entry and statistical analysis were carried out by the statistical package for the social science, version 19. Data for continuous variables were expressed as mean and SD. The categorical variables were expressed in absolute numbers and in percentages. The comparison between two groups was analyzed by a nonparametric test of the Mann-Whitney test for continuous variables; and $\chi^{2}$ for qualitative data. Correlations were performed using the Spearman bivariate correlation. A $P$ value less than 0.05 was considered statistically significant. Separate receiver operating characteristics (ROCs) were generated for the CFF test and MHE detection. The best threshold was defined on the basis of ROC analysis by identifying the $\mathrm{CFF}$ value that gave the best combination of sensitivity and specificity, that is, the value that maximized the sum of the sensitivity and specificity. The analysis of the ROC curve was carried out using version 7.50 of the MedCalc software (Mariakerke, Belgium).

\section{Results}

Our case-control study recruited 100 patients with known chronic liver disease $(77 \% \mathrm{HCV}, 17 \% \mathrm{HBV}$, and $6 \%$ coinfection) and age and sex matched individuals were included as a control group; the
Table 1 Demographic characteristics of the screened drivers

\begin{tabular}{lc}
\hline Variables & Patients $[n(\%)]$ \\
\hline Age (mean \pm SD) & $38.31 \pm 10.36$ \\
Duration of liver disease (mean \pm SD) (years) & $7.83 \pm 3.8$ \\
Sex & \\
$\quad$ Male & $93(93)$ \\
Female & $7(7)$ \\
Residence & \\
$\quad$ Rural & $67(67)$ \\
Urban & $33(33)$ \\
Education & \\
Literate & $41(41)$ \\
Illiterate & $59(59)$ \\
Smoking habit & \\
Smoker & $69(69)$ \\
Nonsmoker & $31(31)$ \\
Driving history & \\
Good driving history & $82(82)$ \\
Bad driving history & $18(18)$ \\
\hline
\end{tabular}

demographic characteristics of the case group are shown in Table 1. A total of 40 patients showed evidence for MHE; however, for 60 patients, no evidence for MHE could be identified. The clinical and biochemical characteristics of the studied patients are shown in Table 2. There were significant differences in SDT, NCT, and CFF $(P<0.001)$ between cases and the control group (Table 3$)$.

Psychometric tests (SDT and NCT-A) were abnormal in $12 \%$, normal in $29 \%$, and not carried out in $59 \%$ of screened drivers, although CFF test was abnormal in $36 \%$ and normal in $64 \%$ of screened drivers. An overall $50 \%$ of patients with MHE were Child A and 50\% were Child B and Child C, while those without MHE (73\%) were Child A and $27 \%$ were Child B and Child C, with a statistically significant difference $(P=0.027)$. The abnormal CFF test was significantly common among patients with Child C (69.2\%), while there was no significant difference of results of SDT and NCT among different Child grades (Table 4). There was significant reverse correlation between the CFF score and the Child-Pugh score ( $r=-0.271, P=0.030)$ (Fig. 1).

The sensitivity and specificity of ROC curves for CFF in the diagnosis of MHE was $100 \%$, with a cutoff of less than or equal to $38.5 \mathrm{~Hz}$, and the area under the curve was 1.00 (95\% confidence interval, 0.964-1.00) $(P<0.0001)$ (Fig. 2).

\section{Discussion}

MHE negatively affects quality of life related to health (HRQOL); complex activities including attention, information processing, and psychomotor expertise 
Table 2 Clinical characteristics of the screened drivers according to presence and absence of minimal hepatic encephalopathy

\begin{tabular}{|c|c|c|c|}
\hline Parameters & $\begin{array}{l}\text { MHE-positive } \\
\text { group }(N=40)\end{array}$ & $\begin{array}{l}\text { MHE-negative } \\
\text { group }(N=60)\end{array}$ & $\begin{array}{c}P \\
\text { value }\end{array}$ \\
\hline Age & $51.72 \pm 7.97$ & $46.03 \pm 11.18$ & 0.016 \\
\hline $\begin{array}{l}\text { Duration of liver } \\
\text { disease (years) }\end{array}$ & $9.18 \pm 3.9$ & $6.9 \pm 3.5$ & 0.003 \\
\hline Sex (male/female) & $38 / 2$ & $55 / 5$ & 0.552 \\
\hline $\begin{array}{l}\text { Education (literate/ } \\
\text { illiterate) }\end{array}$ & $20 / 20$ & $23 / 37$ & 0.248 \\
\hline Smoking & 29 & 40 & 0.537 \\
\hline $\begin{array}{l}\text { Residence (rural/ } \\
\text { urban) }\end{array}$ & $28 / 12$ & $39 / 21$ & 0.602 \\
\hline Bad driving history & 18 & 0 & $<0.001$ \\
\hline ALT (U/I) & $46.80 \pm 24.73$ & $48.20 \pm 28.36$ & 0.720 \\
\hline AST (U/I) & $49.55 \pm 25.13$ & $53.13 \pm 32.70$ & 0.978 \\
\hline $\begin{array}{l}\text { Serum bilirubin } \\
(\mu \mathrm{mol} / \mathrm{l})\end{array}$ & $32.98 \pm 20.18$ & $21.88 \pm 16.30$ & 0.004 \\
\hline $\begin{array}{l}\text { Serum albumin } \\
(\mathrm{mg} / \mathrm{dl})\end{array}$ & $33.69 \pm 7.16$ & $38.69 \pm 6.53$ & 0.001 \\
\hline $\begin{array}{l}\text { Serum sodium } \\
(\mathrm{mmol} / \mathrm{l})\end{array}$ & $138.42 \pm 2.64$ & $138.53 \pm 3.45$ & 0.618 \\
\hline $\begin{array}{l}\text { Serum potassium } \\
(\mathrm{mmol} / \mathrm{l})\end{array}$ & $4.1 \pm 0.34$ & $4.1 \pm 0.41$ & 0.850 \\
\hline INR & $1.57 \pm 0.65$ & $1.28 \pm 0.49$ & 0.044 \\
\hline Prothrombin time & $14.82 \pm 3.1$ & $12.99 \pm 2.4$ & 0.001 \\
\hline $\begin{array}{l}\text { Prothrombin } \\
\text { concentration }\end{array}$ & $77.28 \pm 17.48$ & $84.47 \pm 16.47$ & 0.018 \\
\hline Hemoglobin (g \%) & $12.77 \pm 1.47$ & $13.07 \pm 1.26$ & 0.189 \\
\hline $\begin{array}{l}\text { Platelet count } \\
\left(\times 10^{3} / \mathrm{mm}^{3}\right)\end{array}$ & $183.60 \pm 63.93$ & $181.48 \pm 69.63$ & 0.808 \\
\hline CFF test $(\mathrm{Hz})$ & $36.83 \pm 5.24$ & $42.26 \pm 1.79$ & $<0.001$ \\
\hline
\end{tabular}

Values are presented as mean $\pm \mathrm{SD}$. ALT, alanine aminotransferase; AST, aspartate aminotransferase; CFF, critical flicker frequency; INR, international normalized ratio; MHE, minimal hepatic encephalopathy.

such as car driving and planning a trip are mainly affected. However, the basic activities of daily life, such as shopping, clothing, and personal cleanliness, are well preserved. Given the high prevalence of liver diseases in Egypt, early identification of people at risk is of outmost importance, as management of $\mathrm{MHE}$ would improve the psychometric performance and quality of life, and can terminate many occupational risks, including road accidents.

In this study, a self-reported driving history with a statistically significant number of traffic offenses was obtained from the screened drivers with MHE (45\%) compared with non-MHE patients $(0 \%)(P<0.001)$. Over 5 years' of data analysis has shown that most of the patients with MHE (53\%) reported traffic car accidents or violations in comparison with $23 \%$ of patients without MHE [11]. Gad et al. [9] also reported that $33 \%$ of patients with $\mathrm{MHE}$ reported a traffic accident or violation within the former year when compared with only $4 \%$ of patients without MHE. Wein et al. [12] showed that patients with
Table 3 Comparison between cases and controls with regard to psychometric tests and critical flicker frequency tests

\begin{tabular}{lccc}
\hline & Cases $(N=100)$ & Control $(N=30)$ & $P$ value \\
\hline NCT $($ mean $\pm S D)$ & $66.85 \pm 43.58$ & $32.13 \pm 11.66$ & $<0.001$ \\
SDT $($ mean $\pm S D)$ & $76.90 \pm 30.26$ & $28.79 \pm 18.33$ & $<0.001$ \\
CFF test $($ mean $\pm S D)$ & $40.09 \pm 4.46$ & $45.53 \pm 2.85$ & $<0.001$ \\
\hline
\end{tabular}

CFF, critical flicker frequency; NCT, number connection test; SDT, symbol digits test.

Table 4 Relationship between the critical flicker frequency test and psychometric tests and the Child-Pugh grade

\begin{tabular}{lcccc}
\hline & $\begin{array}{l}\text { Child A } \\
(N=64) \\
{[-n(\%)]}\end{array}$ & $\begin{array}{l}\text { Child B } \\
(N=23) \\
{[n(\%)]}\end{array}$ & $\begin{array}{c}\text { Child C }(N=13) \\
{[n(\%)]}\end{array}$ & $\begin{array}{c}P \\
\text { value }\end{array}$ \\
\hline $\begin{array}{l}\text { Abnormal } \\
\text { SDT }\end{array}$ & $5(7.8)$ & $2(8.7)$ & $5(38.5)$ & 0.115 \\
$\begin{array}{l}\text { Abnormal } \\
\text { NCT }\end{array}$ & $5(7.8)$ & $2(8.7)$ & $5(38.5)$ & 0.115 \\
$\begin{array}{l}\text { Abnormal } \\
\text { CFF test }\end{array}$ & $16(25)$ & $11(47.8)$ & $9(69.2)$ & 0.004 \\
\hline
\end{tabular}

CFF, critical flicker frequency; NCT, number connection test; SDT, symbol digits test.

MHE had reduced driving performance in a 90-min standardized road driving test. They had worse ratings in car handling categories, adaptation maneuver, and caution compared with patients without MHE. Therefore, MHE not only harms drivers' work performance but also has the potential to threaten life. For example, serious driving offenses for nonalcoholic drivers in countries where liver disease is endemic would alert clinicians to consider MHE as an important diagnostic option. In a recent study by Kircheis et al. [13], patients with HE overestimated their driving skills despite significant driving deficits.

MHE is more evident with the progress of liver fibrosis and advancement of Child's score [14]. In our study, the proportion of patients with MHE increased with the increase in the Child-Pugh grade as follows: 20 of $64(31.1 \%)$ patients with compensated liver cirrhosis (Child-Pugh grade A), 11 of 23 (47.8\%) patients with decompensated liver cirrhosis (Child-Pugh grades B), and nine of $13(69.2 \%)$ patients with Child-Pugh grades $\mathrm{C}(P=0.008)$. This finding is consistent with those of previous studies [15-18]. Our result showed significant reverse correlation between $\mathrm{CFF}$ test score and Child-Pugh score $(r=-0.271, \quad P=0.030)$. In agreement with us, the Manuel et al. study [15] reported that CFF correlated weakly with Child-Pugh score in patients with cirrhosis ( $r=-0.22, P=0.018$ ). Furthermore, the Sharma et al. study [10] showed a significant correlation between $\mathrm{CFF}$ and Child-Pugh score. However, there was no difference in prevalence of MHE according to Child-Pugh status. On multivariate regression, 


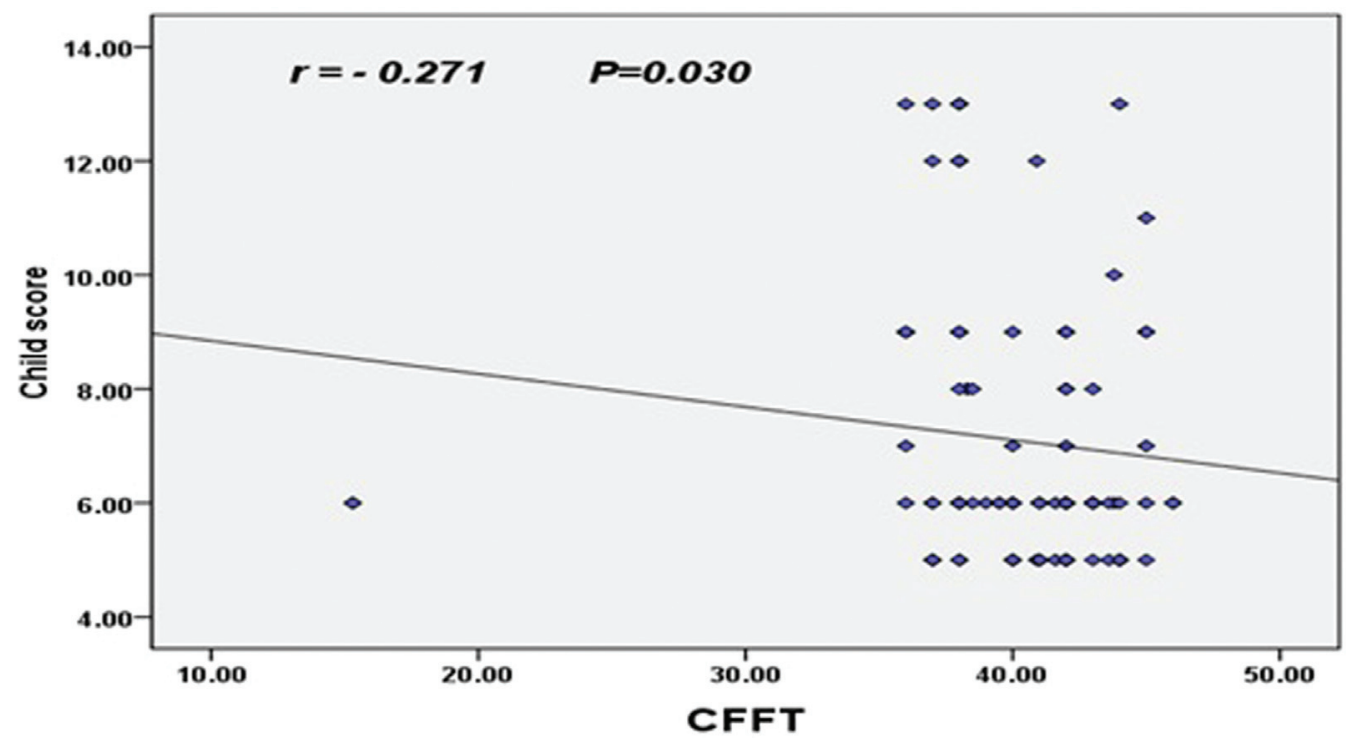

Correlation between CFF test and Child-Pugh score. CFF, critical flicker frequency.

Figure 2

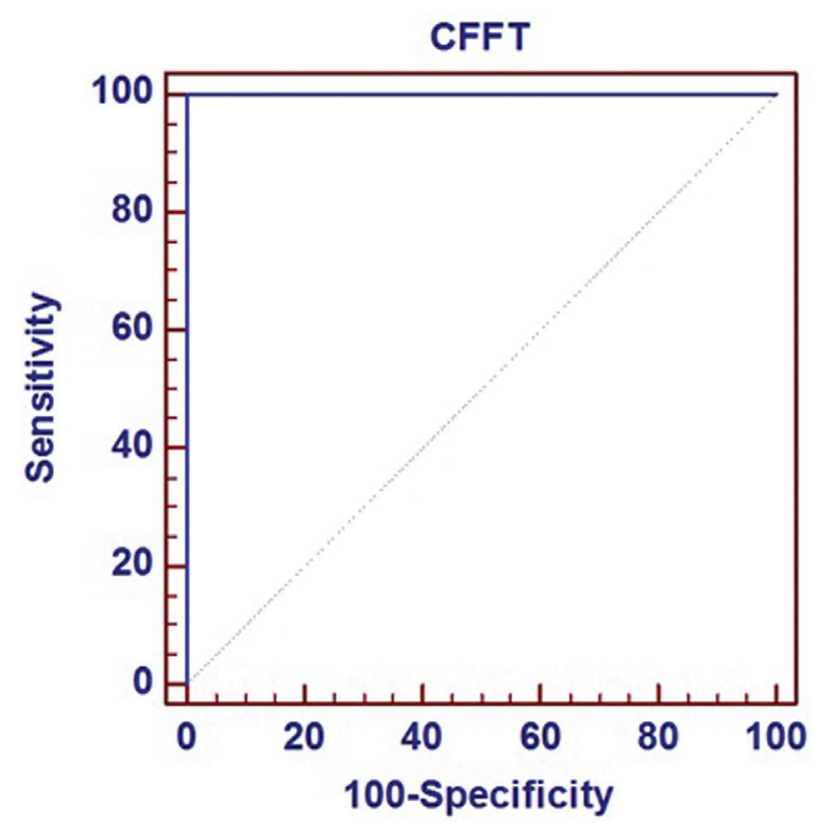

Shows receiver operating characteristic curves for sensitivity and specificity of CFF test in the diagnosis of MHE with a cutoff of less than or equal to $38.5 \mathrm{~Hz}$; the sensitivity was $100 \%$ and specificity was $100 \%$. The area under the curve was 1.00 (95\% confidence interval, 0.964-1.00) $(P<0.0001)$. CFF, critical flicker frequency; MHE, minimal hepatic encephalopathy.

patients admitted due to road accidents, with liver cirrhosis and age more than 65 years, have a significantly higher mortality [19]. A combination of bleeding disorders, sepsis, and hepatic dysfunction has been noted as contributing factors to this worse prognosis [20].Age and educational status are recognized widely as being associated to neuropsychological test results and therefore normal values of healthy controls for age and education are recommended [5]. In our study, there was a significant difference in age between those with and without MHE. In the study by $\mathrm{Li}$ et al. [17], no difference was found in age between patients with and without MHE.

\section{Conclusion}

Our data revealed a high prevalence of MHE (40\%) among Egyptian drivers with liver cirrhosis. MHE diagnosed by psychometric tests was $12 \%$ in screened drivers with cirrhosis in comparison with $36 \%$ diagnosed by CFF. Hence, we conclude that $\mathrm{CFF}$ is a simple, reliable tool for the diagnosis of MHE with no age or literacy requirement. Therefore, we suggested including the CFF test in screening of drivers with chronic liver disease for early discovery and proper management, as a value of CFF less than or equal to the cutoff of $39 \mathrm{~Hz}$ indicates a diagnosis of MHE.

\section{Financial support and sponsorship \\ Nil.}

\section{Conflicts of interest}

There are no conflicts of interest.

\section{References}

1 Vilstrup H, Amodio P, Bajaj J, Cordoba J, Ferenci P, Mullen KD, et al. Hepatic encephalopathy in chronic liver disease: 2014 Practice Guideline by the American Association for the Study of Liver Diseases and the European Association for the Study of the Liver. Hepatology 2014; 60:715-735.

2 Quero JC, Hartmann IJ, Meulstee J, Hop WC, Schalm SW. The diagnosis of subclinical hepatic encephalopathy in patients with cirrhosis using neuropsychological tests and automated electroencephalogram analysis. Hepatology 1996; 24:556-560. 
3 Schomerus H, Hamster W, Blunck H, Reinhard U, Mayer K, Dolle W. Latent portasystemic encephalopathy. I. Nature of cerebral functional defects and their effect on fitness to drive. Dig Dis Sci 1981; 26:622-630.

4 Bajaj JS, Etemadian A, Hafeezullah M, Saeian K. Testing for minimal hepatic encephalopathy in the United States: an AASLD survey. Hepatology 2007; 45:833-834.

5 Ferenci P, Lockwood A, Mullen K, Tarter R, Weissenborn K, Blei AT. Hepatic encephalopathy-definition, nomenclature, diagnosis, and quantification: final report of the working party at the 11th World Congresses of Gastroenterology, Vienna, 1998. Hepatology 2002; 35:716-721.

6 Randolph C, Hilsabeck R, Kato A, Kharbanda P, Li YY, Mapelli D, et al. Neuropsychological assessment of hepatic encephalopathy: ISHEN practice guidelines. Liver Int 2009; 29:629-635.

7 Weissenborn K, Heidenreich S, Ennen J, Ruckert N, Hecker H. Attention deficits in minimal hepatic encephalopathy. Metab Brain Dis 2001; 16:13-19.

8 Sharma K, Pant S, Misra S, Dwivedi M, Misra A, Narang S, et al. Effect of rifaximin, probiotics, and I-ornithine l-aspartate on minimal hepatic encephalopathy: a randomized controlled trial. Saudi J Gastroenterol 2014; 20:225-232.

9 Gad YZ, Zaher AA, Moussa NH, El-desoky AE, Al-Adarosy HA. Screening for minimal hepatic encephalopathy in asymptomatic drivers with liver cirrhosis. Arab J Gastroenterol 2011; 12:58-61.

10 Sharma P, Sharma BC, Puri V, Sarin SK. Critical flicker frequency: diagnostic tool for minimal hepatic encephalopathy. J Hepatol 2007; 47:67-73.

11 Bajaj JS, Hafeezullah M, Hoffmann RG, Saeian K. Minimal hepatic encephalopathy: a vehicle for accidents and traffic violations. Am J Gastroenterol 2007; 102:1903-1909.
12 Wein C, Koch H, Popp B, Oehler G, Schauder P. Minimal hepatic encephalopathy impairs fitness to drive. Hepatology 2004; 39:739-745.

13 Kircheis G, Knoche A, Hilger N, Manhart F, Schnitzler A, Schulze H, et al. Hepatic encephalopathy and fitness to drive. Gastroenterology 2009; 137:1706-1715, e1-9.

14 Das A, Dhiman RK, Saraswat VA, Verma M, Naik SR. Prevalence and natural history of subclinical hepatic encephalopathy in cirrhosis. J Gastroenterol Hepatol 2001; 16:531-535.

15 Romero-Gomez M, Cordoba J, Jover R, del Olmo JA, Ramirez M, Rey R, et al. Value of the critical flicker frequency in patients with minimal hepatic encephalopathy. Hepatology 2007; 45:879-885.

16 Wang JY, Zhang NP, Chi BR, Mi YQ, Meng LN, Liu YD, et al. Prevalence of minimal hepatic encephalopathy and quality of life evaluations in hospitalized cirrhotic patients in China. World J Gastroenterol 2013; 19:4984-4991.

17 Li SW, Wang K, Yu YQ, Wang HB, Li YH, Xu JM. Psychometric hepatic encephalopathy score for diagnosis of minimal hepatic encephalopathy in China. World J Gastroenterol 2013; 19:8745-8751.

18 AmpueroJ, Simon M, Montoliu C, Jover R, Serra MA, Cordoba J, etal. Minimal hepatic encephalopathy and critical flicker frequency are associated with survival of patients with cirrhosis. Gastroenterology 2015; 149:1483-1489.

19 Bajaj JS, Ananthakrishnan AN, McGinley EL, Hoffmann RG, Brasel KJ. Deleterious effect of cirrhosis on outcomes after motor vehicle crashes using the nationwide inpatient sample. Am J Gastroenterol 2008; 103:1674-1681.

20 Teh SH, Nagorney DM, Stevens SR, Offord KP, Therneau TM, Plevak DJ, et al. Risk factors for mortality after surgery in patients with cirrhosis. Gastroenterology 2007; 132:1261-1269. 